\title{
Dietary glycaemic index, glycaemic load and risk of reflux oesophagitis, Barrett's oesophagus and oesophageal adenocarcinoma
}

\author{
H. G. Mulholland ${ }^{1}$, M. M. Cantwell ${ }^{1}$, L. A. Anderson ${ }^{1}$, B. T. Johnston ${ }^{2}$, R. G. P. Watson ${ }^{2}$, \\ S. J. Murphy ${ }^{3}$, H. R. Ferguson ${ }^{4}$, J. McGuigan ${ }^{2}$, J. V. Reynolds ${ }^{5}$, H. Comber ${ }^{6}$ and L. J. Murray ${ }^{1}$ \\ ${ }^{1}$ Cancer Epidemiology \& Prevention Research Group, Queens University Belfast, Belfast BT12 6BJ, UK, ${ }^{2}$ Belfast Health \& \\ Social Care Trust, Belfast BT8 8BH, UK, ${ }^{3}$ Daisy Hill Hospital, Belfast BT35 8DR, UK, ${ }^{4}$ Craigavon Area Hospital, Belfast \\ BT63 5QQ, UK, ${ }^{5}$ St James's Hospital, Dublin, Republic of Ireland and ${ }^{6}$ National Cancer Registry, Cork, Republic of Ireland
}

Incidence rates of reflux oesophagitis (RO), Barrett's oesophagus (BO) and oesophageal adenocarcinoma (OAC) appear to be rising in Western communities, particularly among men ${ }^{(1-3)}$. The majority of OAC cases develop as a result of progression from $\mathrm{BO}$, which is often preceded by RO. It is important to identify potentially-modifiable risk factors, such as dietary habits, that may affect the risk of developing RO, BO and OAC. Studies of other cancer sites have suggested a possible association between dietary glycaemic index (GI) and glycaemic load (GL) and cancer risk ${ }^{(4)}$ but this relationship has yet to be explored for OAC.

In an all-Ireland case-control study information was collected from patients with OAC ( $n$ 224), long-segment BO ( $n$ 220), RO ( $n$ 219) and healthy population-based controls ( $n$ 256) on lifestyle factors and dietary intake using a 101-item FFQ between March 2002 and July 2005. Multiple logistic regression analysis was applied to examine the association between dietary GI, GL or total carbohydrate intake and disease risk, comparing the highest $v$. lowest tertiles of intake and also using continuous variables. Regression models adjusted for confounding variables including age, gender, energy intake, smoking, education, occupation, alcohol, medication use and gastrooesophageal reflux symptoms. The analyses were also conducted based on stratification by BMI and waist:hip ratio categories.

There were no significant associations between total carbohydrate, GI or GL intake and RO or BO. OAC risk was significantly reduced for each $50 \mathrm{~g} / \mathrm{d}$ increase in total carbohydrate intake (OR 0.46 (95\% CI 0.33, 0.63)), which remained across all BMI and waist:hip ratio categories. However, a significant increased risk of OAC was detected for each 10 unit increment in GI intake (OR 1.36 (95\% CI 1.01 , 1.82)), which was further increased among overweight individuals with a high waist:hip ratio (OR 1.86 (95\% CI 1.20, 2.90)). No significant associations were observed between GL and OAC risk.

These findings indicate that a high GI intake is positively associated with the risk of OAC, particularly among centrally-overweight individuals, and may have contributed to the rising incidence rates. However, total carbohydrate intake was inversely associated with OAC risk. These findings suggest that individuals should eat a diet that is high in carbohydrate content and choose low-GI carbohydrates where possible to reduce their risk of developing OAC.

1. Bosetti C, Levi F, Ferlay J, Garavello W, Lucchini F, Bertuccio P, Negri E \& La Vecchia C (2008) Int J Cancer 122, 1118-1129.

2. Fitzgerald RC (2004) Aliment Pharmacol Ther 20, Suppl. 8, S45-S49.

3. Lassen A, Hallas J \& de Muckadell OB (2006) Am J Gastroenterol 101, 1193-1199.

4. Gnagnarella P, Gandini S, La Vecchia C \& Maisonneuve P (2008) Am J Clin Nutr 87, 1793-1801. 